\title{
The Numerical Approach to Analysis of Microchannel Cooling Systems
}

\author{
Ewa Raj, Zbigniew Lisik, Malgorzata Langer, \\ Grzegorz Tosik, and Janusz Wozny \\ Institute of Electronics, Technical University of Lodz, \\ 223 Wolczanska Str, 90-924, Lodz, Poland \\ \{ewaraj, lisikzby, malanger, \\ grzegorz.tosik, jwozny\}@p.lodz.pl
}

\begin{abstract}
The paper deals with microchannel cooling where water is the cooling liquid. ANSYS software and CFDRC-ACE one were used to analyse the flows and the origin of large amount of heat that can be overtaken from the chip when microchannels are applied. The concept of microscale heat transfer coefficient is discussed. The phenomena taking place in microchannel flows are simulated and some conclusions are introduced to explain the results met in many references but still unexplained. In contrast to existing, standard methods, the new approach describes the local phenomena and is used in the further investigation of the cooling microstructure. An effect of its geometry on the total overtaken heat flux is analysed with respect to optimal conditions as well as to technological restrictions.
\end{abstract}

\section{Introduction}

The fast development of nowadays electronics induces the increase of heat dissipation inside semiconductor structures. In case of power module, the total power can exceed $1 \mathrm{~kW}[1,2]$ and the heat flux that needs to be overtaken from the device reaches several $\mathrm{MW} / \mathrm{m}^{2}$. The greatest problem nowadays is not only the huge amount of heat dissipation but mainly its density at the surface of the structure. Therefore, one has revealed the challenging task: to design as effective heat exchanger as only possible with regard to microelectronic dimensions restrictions.

A forced cooling system with the coolant characterised by the large enough heat capacity and thermal conductivity could meet these demands only. For example, it could be a liquid cooling system with water as a cooling medium, and such systems already exist. Their effectiveness can be improved when one introduces the coolant stream as close to the heat source as possible. This idea has been employed in a new solution of a liquid cooling system that is based on microchannels formed in the substrate of semiconductor device [3-5], or in cooling microstructure placed at the chip directly [6, 7]. Unfortunately, such a huge heat transfer capacity has been observed at very large inlet pressure, e.g. above $200 \mathrm{kPa}$ in [3], that is unacceptable because of the reliability and the life of the electronic equipment.

Although the differences in the behaviour of liquid flow in the micro- and macrostructures have been already reported [3-12], no coherent explanation of their 
origin exists. In [11], they have noticed that the flow character in microchannels changes for much smaller values of Reynolds numbers. Others observed in [12] the slip of water flowing in microchannels while there is no possibility to observe the phenomenon in macrochannels under these particular conditions. It indicates that the transition from the macroscale, corresponding to the typical liquid cooling systems, to the microscale, when the considered thermal phenomena take place, is not the scaling problem only but it creates quite new problems in heat and mass transport. The lack of their satisfactory description as well as the contradictory opinions presented by different authors encouraged us to analyse the problems from the microchannel cooling efficiency aspect. Some of our results are presented in the paper.

\section{Heat and Mass Transfer in Microchannels}

The rise of turbulent flow should be described from the origin; when the fluid makes contact with the surface, viscous effects become significant. As a result, boundary layers develop with increasing distance from the inlet, until they merge at the centreline. Then the flow is fully developed and the distance at which this condition is achieved is called hydrodynamic entrance length. Within the fully developed region one can distinguish a turbulent core and a laminar sublayer. In the first region the heat is transferred by the mass transport whereas in the second one, the heat transport is dominated by diffusion. In consequence, this area is responsible for heat exchange at the solid-fluid border mainly.

\subsection{One Channel Approach}

At first, our interest has been devoted to analyse the heat overtaking process by the water flow through a single channel. We have based it on simulations of water cooling conducted with the aid of ANSYS and CFDRC-ACE software. The heat transfer process has been investigated with use of the heat transfer coefficient that represents the amount of heat overtaken by the coolant that flows along the cooled walls with respect to assumed temperature difference between the wall temperature and the reference ambient one. When one considers liquid heat exchange systems, the heat transfer coefficient is commonly considered as the ratio of the heat flux overtaken from the wall at some particular point and the difference of the point temperature and some reference one that usually is the liquid inlet temperature, the same for the whole heat exchange system [13]. Such an attitude to the analysis of heat exchange problems can be called the macroscale one with the macroscale heat transfer coefficient that refers the local thermal phenomena to one arbitrary, defined temperature. This feature of the macroscale coefficient allows to treat the system as the whole and to estimate the influence of the system design on the entire heat exchange process. Its value does not, however, depend on the real heat exchange ability at the particular point only and does not characterise the local heat exchange phenomenon. It is evident when we consider the changes of the macroscale coefficient along the channel shown in Fig.1. Its value decreases although no changes in the mechanics of the heat exchange take place. 


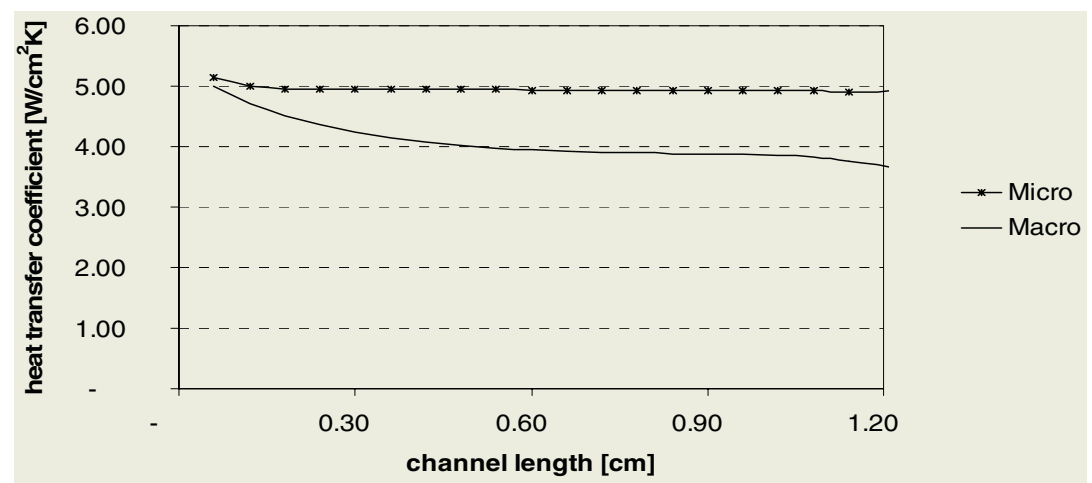

Fig. 1. Micro and macro heat transfer coefficient versus the channel length

The macroscale approach is not sufficient in case of microscale problems, like the microchanel cooling. Therefore, another definition of the heat transfer coefficient, called microscale one, has been proposed. It introduces a new reference temperature connected directly with the local heat exchange process instead of the inlet temperature. It seems that the temperature at the turbulent core - the laminar sublayer border meets this demand the best. The efficiency of the heat overtaking process depends on the heat diffusion that depends directly on the laminar sublayer thickness. The thinner laminar sublayer the smaller difference between the wall temperature and the temperature just on the border, and in consequence the better cooling abilities are. The right of above argument has been confirmed by the results of single channel simulations performed for homogeneous heat dissipation on one wall (Fig.1). While the macroscale coefficient changes along the channel the microscale one remains constant what is in agreement with the mechanics of the heat exchange phenomenon. Its a little larger value at the inlet is obvious when we take into account the laminar sublayer that starts to create itself at the inlet. At the beginning it is very narrow and achieves the final thickness on some distance. At the inlet the laminar sublayer is narrower what leads to more intensive heat exchange.

\subsection{Multi-channel Approach}

In Fig. 2, one can find the outline of the copper structure that has been investigated as an example of multichannel cooler $[8,9]$. It contains several microchannels, with the dimensions $\mathrm{w}_{\mathrm{CH}} \mathrm{xh}$; separated by the walls (columns) of the thickness $\mathrm{w}_{\mathrm{COL}}$. Since the heat exchange processes are homogeneous along the channel if the microscale heat transfer coefficient is used, the $3 \mathrm{D}$ analysis can be simplified to the investigation of $2 \mathrm{D}$ model that is a crosscut in the direction perpendicular to the water flow.

Once again the incoherent reports $[3,5,7]$ forced us to look for the optimal geometry of the presented above structure. The series of numerical simulations lead us to the conclusion that in the microstructure, one can find two competitive phenomena: the enhancement and the chocking effect. The total heat removal increases when the number and the height of channels increase since the larger area of the water-heat sink contact is observed. On the other hand, the overtaken heat flux 
decreases when the column width decreases due to the chocking effect that results in reduction of the temperature at the bottom wall of the channel.

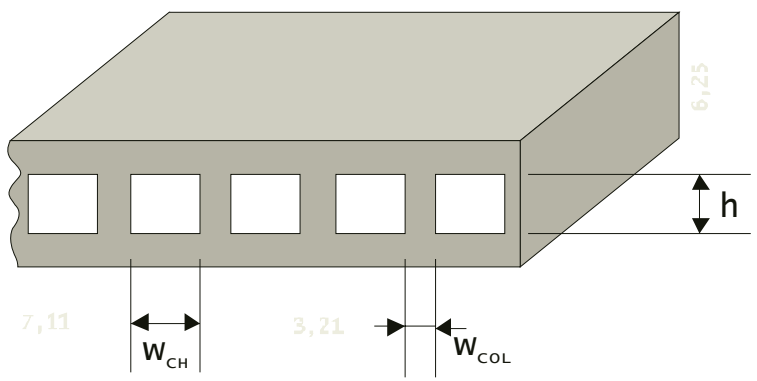

Fig. 2. The 3D multichannel structure

With the aid of numerical analysis, an influence of the channel width and height, the column width as well as the heat transfer coefficient on total heat overtaken from the structure have been examined. The considerations above are illustrated graphically in Fig. 3. The chart presents two curves for the constant column and channel width $(2 \mathrm{~mm})$ and for two chosen heights, one twice as high as the other. The simulations have been performed for the heat transfer coefficient equal to $10 \mathrm{~W} / \mathrm{cm}^{2} \mathrm{~K}$. This value has been settled with the aid of one channel simulations. One can easily notice the peaks in the curves that are created by the two phenomena. The most crucial conclusion is that the optimal geometry of the structure from Fig. 2 exists. There are certain ranges of parameters deviations from the most preferable values where changes have no influence on the efficiency of the whole system.

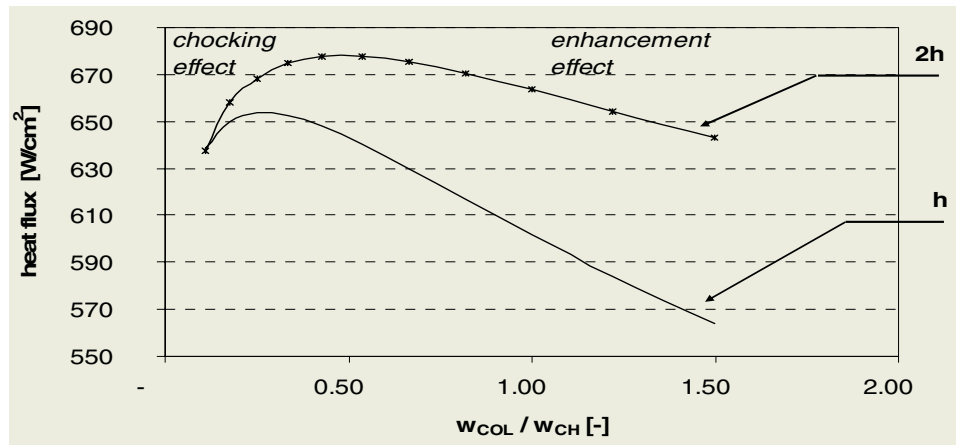

Fig. 3. Two competitive phenomena in the characteristic of heat flux versus the ratio of the column and the channel

\section{Inlet Elements}

All the above considerations deal with the fully developed turbulent flow inside the microchannel cooler. Its efficiency depends, however, on the flow velocity inside the 
channels and the input pressure that are strongly combined each with the other. Since the pressure across the liquid cooling unit is limited in microelectronic applications the pressure losses in inlet elements are of large importance for the final effectiveness of the microchannel coolers. In addition, too high values of pressure and subpressure can be very destructive, shorten the life and lower the reliability of the whole system. Therefore, one must take into considerations the hydrodynamic entrance phenomena.

In this chapter some results of the numerical investigations of the inlet element shape and its influence on the pressure distribution in a microchannel cooler are reported. They present the $2 \mathrm{D}$ analysis that has been performed for the cross-section of the cooling structure shown in Fig.3. It corresponds to the water flow path that consists of the inlet chamber, the contraction and the microchannel section. In Fig. 3 two considered solutions of the cooling structure are depicted - the dotted line in the contraction section shows the sharp edges structure, while the continuous one presents the smooth structure. During the simulations, the contraction length, $d_{k}$, has varied in the range $5 \div 20 \mathrm{~mm}$. The other dimensions have been settled on the basis of the design and technological restrictions as well as the results of earlier numerical analysis. The simulations have been conducted with the aid of ANSYS software for similar boundary conditions as the previous ones. The inlet pressure has been assumed as equal to $40 \mathrm{kPa}$ and the outlet pressure has been kept at $0 \mathrm{~Pa}$. It has been established on the basis of earlier considerations of mass flow resulting from the pump efficiency and additional limits for allowable pressure in microstructures.

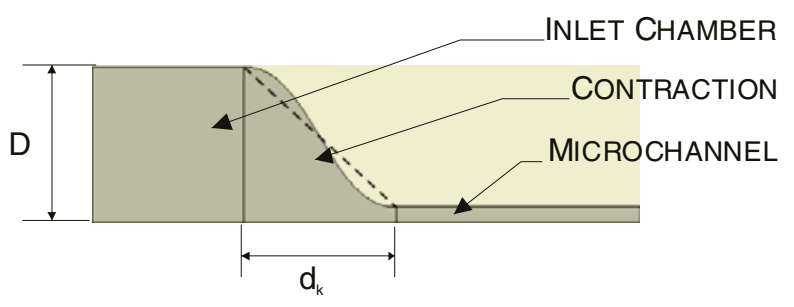

Fig. 4. Outline of test structure

The results presented here concern simulations performed for the both types of structures and for three contraction lengths 5,10 and $20 \mathrm{~mm}$, respectively. They were delivered by ANSYS in a form of maps presenting pressure distribution like the ones in Fig. 5. The figure shows the pressure distribution in the both types of analyzed structures for the contraction length $\mathrm{d}_{\mathrm{k}}=10 \mathrm{~mm}$.

The basic data characterising the hydrodynamics processes in the considered inlet elements, like the highest static subpressure $\mathrm{p}_{\min }$ and the highest static pressure $\mathrm{p}_{\max }$, have been extracted from the pressure maps and are collected in Table 1 together with additional characteristic parameters discussed below.

Comparing the values of maximal and minimal pressure, one can notice a surprising large magnitude of the subpressure in the sharp edge design contrary to the smooth edge one, where the subpressure area almost does not exist. 

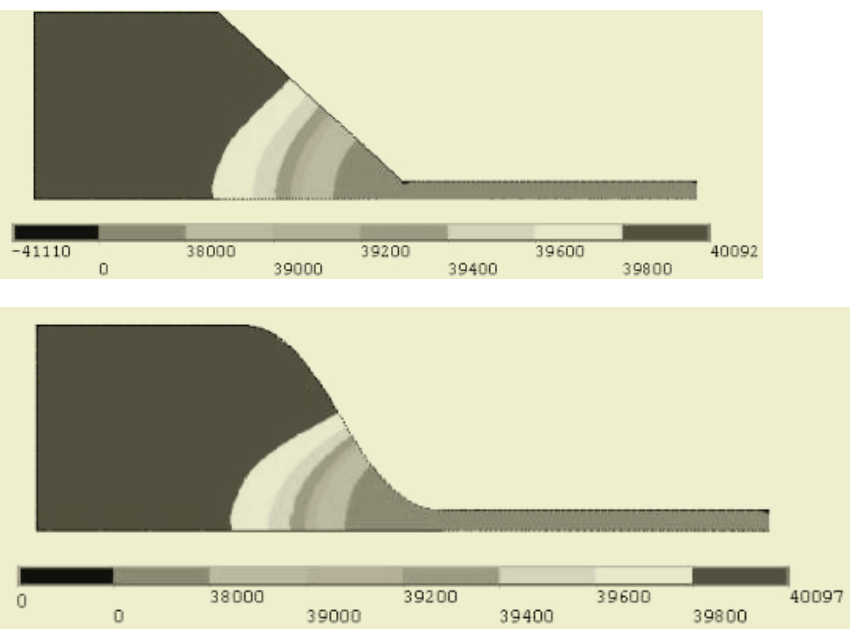

Fig. 5. Pressure distribution in the structures with $d_{k}=10 \mathrm{~mm}$ and with (a) sharp edges and (b) smooth edges

Table 1. Characteristic parameters for various inlet structures

\begin{tabular}{ccccc}
\hline contraction length $\mathrm{d}_{\mathrm{k}}$ & $\mathrm{p}_{\min }[\mathrm{kPa}]$ & $\mathrm{p}_{\max }[\mathrm{kPa}]$ & $\mathrm{p}_{3 \mathrm{~mm}}[\mathrm{kPa}]$ & $\mathrm{d}_{\mathrm{opt}}[\mathrm{mm}]$ \\
\hline \multicolumn{5}{c}{ sharp edge structures } \\
\hline $5 \mathrm{~mm}$ & -47.4 & 40.09 & 4.05 & 6.50 \\
$10 \mathrm{~mm}$ & -41.1 & 40.09 & 5.39 & 3.00 \\
$20 \mathrm{~mm}$ & -20.2 & 40.09 & 5.86 & 1.50 \\
& smooth edge structures & & \\
$5 \mathrm{~mm}$ & -1.8 & 40.11 & 5.92 & 1.40 \\
$10 \mathrm{~mm}$ & 0 & 40.10 & 5.96 & 1.20 \\
$20 \mathrm{~mm}$ & 0 & 40.09 & 5.82 & 1.00 \\
\hline
\end{tabular}

It indicates that the additional efforts to get the smooth edge contraction are worth to be undertaken. It can result in the higher reliability and the considerably longer microstructure life. One can strengthen this result increasing the contraction length $\mathrm{d}_{\mathrm{k}}$. There are some natural limits arisen from the permissible length of the cooling structure but they apply to sharp designs rather. In case of smooth inlets the subpressure becomes negligible small at $\mathrm{d}_{\mathrm{k}}=10 \mathrm{~mm}$. The pressure gradients generated in contraction segment penetrate the entrance of the microchannel disrupting the pressure distribution in that region and changing the heat transfer process inside the whole microstructure. Therefore, it is impossible to use an input microchannel pressure to evaluate the microchannel cooling efficiency. One needs the 
input pressure independent on the local disturbance generated by the contraction segment for this goal. One has assumed that this condition is met at the place where the pressure starts to change linearly and the velocity profile is symmetrical. This distance measured from the microchannel input is denoted as $d_{\text {opt }}$, and its values for considered structures are gathered in Table 1. They depend on the contraction shape and dimensions. The smaller the value the lower turbulence and subpressure occur in the microchannel entrance. In order to evaluate the influence of contraction segment design on the phenomena in the microchannel cooling part, an optimal distance for microchannel pressure determination for all the structures should be settled. It has been chosen as equal to $3 \mathrm{~mm}$ on the base of the $\mathrm{d}_{\text {opt }}$ values. Such a value allows comparing the pressures of all smooth and sharp edge structures. The average values of the static pressure at $3 \mathrm{~mm}$ are gathered in Table 1.

One can notice that the highest values of $\mathrm{p}_{3 \mathrm{~mm}}$ occur for smooth structures. It means that in case of smooth structures one can observe lower pressure drop on inlet element and higher in the microchannel. As a result, the water velocity is higher in the microchannel, the turbulence is more intense and the heat overtaking efficiency is better. On the other hand, the sharp edges introduce strong turbulent eddies at the entrance to the channel that are suppressed at quite a long distance. This kind of turbulences result in the high subpressure and can be very destructive for microchannel structure what is an additional disadvantage of this design contrary to the smooth one. The av. pressure at the $3 \mathrm{~mm}$ from the inlet vs. contraction length for the smooth structure is graphically presented in Fig. 3. The maximum pressure is obtained for $d_{k}=8 \mathrm{~mm}$. For the values of contraction length in the range from 5 to $10 \mathrm{~mm}$, the differences in pressure are lower than $1 \%$. Hence one can choose an arbitrary $\mathrm{d}_{\mathrm{k}}$ value from the given set. An area of low eddy turbulences (subpressure) is created even for the smooth but too abrupt contraction. The elongation of $\mathrm{d}_{\mathrm{k}}$ causes the shrinkage and finally diminishing of the subpressure region. It results in a peak at the curve presented in Fig. 3.

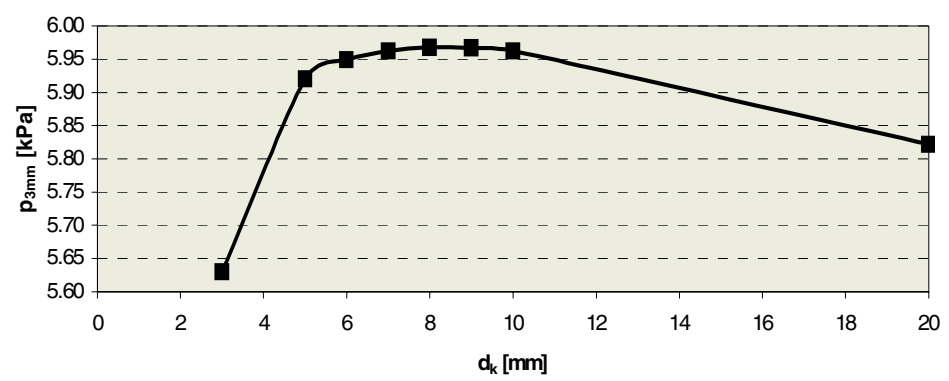

Fig. 6. The average pressure at the $3 \mathrm{~mm}$ for smooth edge structures

\section{Conclusions}

The paper aims at the problem of microchannel liquid cooling system analysis. As an necessary element of the analysis, a new method for heat transfer coefficient 
calculation that is dedicated for microscale analysis has been introduced. Contrary to existing, standard methods, the new approach describes the local phenomena and is used in the further investigation of the cooling microstructure. An effect of its geometry on the total overtaken heat flux is analysed with respect to optimal conditions as well as to technological restrictions. Furthermore, an influence of the shape of the inlet element on pressure distribution in the structure is presented. It is proven that the contraction length as well as the edge design is very essential for life and reliability of the whole system and in consequence for thermal efficiency of the microstructure. The main aims of the inlet element optimisation process are to minimise maximum pressure and subpressure values, to reduce the subpressure area and to decrease the pressure drop losses.

\section{References}

1. Capriz, C.: Trends in Cooling Technologies for Power Electronics. Power Electronics. no $1 / 2$ (1999) 22-24

2. Haque, S. et all: Thermal Management of High-Power Electronics Modules Packaged with Interconnected Parallel Plates. SEMI-THERM, San Diego, USA, (1998) p. 111

3. Tuckerman, D.B., Pease, R.F.W.: High Performace Heat Sinking for VLSI. IEEE Electron Devices Lett. EDL-2 (1981) 126-129

4. Goodson, K.: Electroosmotic Microchannel Cooling System for Microprocessor. Electronics Cooling. Vol.8. (2002)

5. Harms, T.M., Kazimierczak, M.J., Gerner, F.M.: Developing Convective Heat Transfer in Deep Rectangular Microchannels. Int. J. Heat Fluid Flow. Vol.20. (1999) 149-157

6. Sittig, R., Steiner, T.:Vision of Power Modules. Proc. Inter. Conf. CIPS 2000, Bermen, Germany (2000) 134-139

7. Gillot, C.H., Bricard, A., Schaeffer, C.H.: Single and Two-phase heat exchangers for Power Electronics. Int. J. Therm. Sci. Vol.39. (2000) 826-832

8. Raj, E., Langer, M., Lisik, Z.: Numerical Studies for Jet Liquid Cooling in Electronics. Proc. Int. Conf. Thermic'2000. Zakopane, Poland (2000) 68-72

9. Langer, M., Lisik, Z., Raj, E.: Optimising of Microchannel Cooling. Proc. Int. Conf. ICSES'2001. Łódź, Poland (2001) 383-387

10. Pfahler, J. et all: Liquid Transport in Micron and Submicron Channels. J. Sensors Actuators. Vol.21. (1990) 431-434

11. Peng, X.F., Peterson, G.P.: Convective Heat Transfer and Flow Friction for Water in Microchannel Structures. Int. J. Heat Mass Transfer . Vol.39. (1996) 2599-2608

12. Tretheway, D.C., Meinhart, C.D.: Apparent Fluid Slip at Hydrophobic Microchannel Walls. Physics of Fluids. Vol.14. (2002)

13. Incropera, F.P., DeWitt, D.P.: Fundamentals of Heat and Mass Transfer. $3^{\text {rd }}$ ed. Wiley-Interscience, New York (1990) 